\title{
Saúde bucal na escola: avaliação do conhecimento dos pais e da condição de saúde bucal das crianças
}

\section{Oral health in school: assessment of parental knowledge and oral health condition of children}

\author{
Cléa Adas Saliba Garbin* \\ Gabriella Barreto Soares* \\ Izabella Maria Martin ${ }^{* * *}$ \\ Artênio José Ísper Garbin ${ }^{* * * *}$ \\ Renato Moreira Arcieri ${ }^{* * * * *}$
}

\section{Resumo}

Objetivo: avaliar a condição de saúde bucal de pré-escolares e o conhecimento dos pais sobre saúde bucal. Sujeitos e método: estudo transversal, analítico, com amostra de 147 pais e seus respectivos filhos, realizado nas escolas municipais de educação básica de Araçatuba, São Paulo. Foi aplicado um questionário com questões sociodemográficas e de conhecimento de saúde bucal; realizado exame clínico bucal nas crianças para verificar o Ceod e o IHO-S. Os dados foram analisados no software SPSS. Resultados: a maioria dos entrevistados era do sexo feminino $(60,5 \%)$ e tinha entre 18 e 34 anos de idade (66\%). Em relação ao grau de instrução, 56,5\% tinham de 10 a 12 anos de estudo e $68,7 \%$ trabalhavam, porém, a maioria apresentava uma renda familiar entre 1 e 2 salários mínimos (51\%), e $18,4 \%$ recebiam bolsa família. A condição de saúde bucal dos filhos era boa, o Ceod teve média de 0,68. A maioria das crianças apresentou um índice de higiene oral médio (1,51 IHO-S, dp=0,48). Na análise bivariada, houve associação entre a causa da cárie dentária e a variável sexo $(p=0,036)$ dos pais; o que é preciso para prevenir a cárie teve associação estatística com a idade ( $p=0,045)$ e a raça $(p=0,008)$ dos pais. Conclusão: o conhecimento dos pais e responsáveis sobre saúde bucal não foi adequado, apesar da boa condição de saúde bucal dos seus filhos. Fatores sociodemográficos como gênero, idade, raça e grau de instrução dos pais estiveram associados a um maior conhecimento sobre saúde bucal.

Palavras-chave: Conhecimento. Educação em saúde. Pré-escolar. Saúde bucal.

\section{Introdução}

Educação em saúde é uma importante ferramenta para levar ao indivíduo mais conhecimento na busca por orientá-lo para mudanças de atitudes e desenvolvimento de hábitos saudáveis ${ }^{1}$. A odontologia tem papel fundamental na prevenção e na promoção de saúde bucal, com ações educativas para crianças, pais, gestores e profissionais de saúde ${ }^{2}$. A melhoria no conhecimento dos pais, como resultado do programa de educação em saúde bucal, tem sido reconhecida não apenas por influenciar as práticas e os comportamentos em relação à saúde bucal das crianças, mas também melhorar os parâmetros clínicos de saúde bucal como higiene oral, saúde gengival e cárie dentária ${ }^{3,4}$.

Professora doutora adjunta do Departamento de Odontologia Infantil e Social da Faculdade de Odontologia, Universidade Estadual Paulista, Araçatuba, São Paulo, Brasil.

Doutoranda do Departamento de Odontologia Infantil e Social da Faculdade de Odontologia, Universidade Estadual Paulista, Araçatuba, São Paulo, Brasil. Aluna de graduação da Faculdade de Odontologia, Universidade Estadual Paulista, Araçatuba, São Paulo, Brasil.

**** Professor doutor adjunto do Departamento de Odontologia Infantil e Social da Faculdade de Odontologia, Universidade Estadual Paulista, Araçatuba, São Paulo, Brasil.

***** Professor doutor adjunto do Departamento de Odontologia Infantil e Social da Faculdade de Odontologia, Universidade Estadual Paulista, Araçatuba, São Paulo, Brasil. 
Muitos estudos têm comprovado que é possível a prevenção e o controle das doenças bucais por meio da modificação de seus fatores etiológicos ${ }^{5}$. Em relação à cárie, as medidas preconizadas para sua prevenção baseiam-se, fundamentalmente, na educação e na motivação do paciente em relação à desorganização da placa bacteriana, à restrição do consumo de açúcar e ao uso do flúor ${ }^{6}$. A educação assume, então, um papel de destaque na conquista de bons níveis de saúde bucal, facilitando o desenvolvimento de uma consciência crítica nos indivíduos sobre as causas de seus problemas, provocando o interesse e a responsabilidade pela manutenção da sua saúde ${ }^{5,7}$.

As doenças bucais estão entre as mais comuns e prevalentes em todo o mundo, e a condição de saúde bucal precária pode ter um impacto significativo na qualidade de vida das crianças, podendo levar à deterioração da saúde geral do indivíduo ${ }^{8}$. No Brasil, a cárie ainda é um importante problema de saúde pública, como constatado no último levantamento epidemiológico realizado pelo Ministério da Saúde em $2010^{9}$. Isso acontece porque essa doença, assim como a doença periodontal, está associada a condições sociais, econômicas, educacionais e políticas, que ultrapassam a questão da higiene bucal ${ }^{10}$.

$\mathrm{O}$ ambiente familiar constitui o primeiro local de aprendizado, no qual a criança passa a maior parte do tempo e constrói as suas características sociais, culturais e educacionais. É a família que passa seus valores, conhecimentos, suas crenças e, inclusive, práticas em relação à saúde ${ }^{10}$. No entanto, a escola proporciona um ambiente ideal para a educação em saúde, em combinação com atividades de prevenção para melhorar a saúde da criança. Em todo o mundo, as escolas têm sido reconhecidas como um ambiente adequado para realizar práticas educativas de forma eficiente e eficaz, pois reúne um número grande de escolares e capacita também os educadores $^{11,12}$

O trabalho educativo com crianças no período escolar é indicado porque é nessa fase que o ser humano está crescendo e se desenvolvendo, tanto física quanto intelectualmente. As crianças, nessa fase, são mais receptivas e aprendem de maneira mais rápida, o que facilita a aquisição de hábitos adequados relacionados à saúde bucal ${ }^{5,13}$. Além disso, as atividades educativas realizadas nas escolas levam conhecimento aos pais de forma indireta, pois seus filhos aprendem e, depois, ensinam em casa, tornando-se agentes multiplicadores de saúde, evitando assim práticas que favoreçam o aparecimento da doença ${ }^{14}$.

Experiências relatam de forma positiva a atuação dos pais na orientação de hábitos saudáveis de saúde dos filhos. Por isso, é importante sabermos em que nível está o conhecimento dos pais a respeito da saúde bucal ${ }^{15}$. Dessa forma, o objetivo do presente estudo foi avaliar o conhecimento dos pais das crianças matriculadas em escolas municipais de educação básica de Araçatuba, São Paulo, e, também, a condição de saúde bucal de seus filhos. Além disso, pretende-se verificar fatores que influenciam no conhecimento sobre saúde bucal dos pais.

\section{Sujeitos e método}

O estudo foi aprovado pelo Comitê de Ética em Pesquisa com Seres Humanos da Faculdade de Odontologia de Araçatuba (FOA) da Universidade Estadual Paulista (Unesp), de acordo com o processo FOA-0649/10, tendo sido realizado com a compreensão e o consentimento por escrito dos pais dos escolares.

Trata-se de um estudo quantitativo exploratório de corte transversal e analítico, realizado em três escolas municipais de educação básica (Emebs) de Araçatuba. Os sujeitos investigados participaram do projeto de extensão de educação em saúde bucal realizado pelo Programa de Pós-Graduação em Odontologia Preventiva e Social da FOA-Unesp.

Participaram do estudo 147 pais ou responsáveis e seus respectivos filhos de 0 a 6 anos que estudavam nas Emebs e que consentiram a participação no estudo. A coleta de dados foi realizada durante seis meses, após um estudo piloto em que todas as variáveis foram testadas. Os dados foram coletados por meio de questionário semiestruturado, com perguntas fechadas, especialmente elaboradas para a pesquisa. As questões propostas abordaram dados sociodemográficos e o conhecimento por parte dos pais sobre saúde bucal.

As variáveis de caracterização sociodemográfica dos pais incluíram: idade, sexo, cor, estado civil, escolaridade, vínculo trabalhista e renda familiar; as questões relacionadas ao conhecimento abordaram: doenças que podem ocorrer na boca, transmissibilidade, etiologia e modo de prevenção da cárie, frequência ideal de escovação, frequência ideal de uso de fio dental, quantidade de creme dental, idade para primeira consulta ao dentista, se dente decíduo cariado deve ser restaurado, se uso de chupeta prolongado é prejudicial e a idade que a criança deve abandonar a chupeta.

Foi realizado exame de saúde bucal com os pré-escolares na própria escola, o qual foi conduzido por pesquisador calibrado. Para realização do exame, foram realizadas sondas recomendadas pela Organização Mundial da Saúde e espelhos bucais. A condição dos dentes foi avaliada de acordo com os códigos e critérios para levantamento epidemiológico da Organização Mundial de Saúde ${ }^{16}$. Para a condição dos dentes, foi utilizado o número médio de dentes cariados, perdidos ou obturados (Ceod). Foi verificada também a higiene bucal da criança, de acordo com o Índice de Higiene Oral - Simplificado (IHO-S), descrito por Greene e Vermillion ${ }^{17}$.

Foram realizadas análises descritivas por meio de medidas de tendência central (frequências simples, médias e medianas) e medidas de dispersão (desvio padrão) para caracterização sociodemográ- 
fica da população e para elucidar o conhecimento dos pais sobre saúde bucal. Para a rejeição ou não de qualquer das hipóteses, foi considerado um nível de significância de 0,05 . Foram realizadas análises bivariadas entre as questões sobre conhecimento de saúde bucal (variáveis dependentes) com os dados sociodemográficos (variáveis independentes), para o que foi utilizado o teste Qui-Quadrado, sendo que, para os resultados menores do que cinco, foi utilizado o teste Exato de Fisher (para tabelas 2x2) ou a razão da máxima verossimilhança nas questões que admitiam mais de duas categorias. Todas as análises estatísticas foram realizadas utilizando o programa SPSS versão 17.0.

\section{Resultados}

Em relação aos responsáveis, a maior parte era do sexo feminino $(60,5 \%)$ e tinha entre 18 e 34 anos (69,4\%). Quanto ao grau de instrução, 56,5\% tinham de 10 a 12 anos de estudo e $68,7 \%$ trabalhavam, porém, a maioria apresentava uma renda familiar entre 1 e 2 salários mínimos (51\%), e 18,4\% recebiam bolsa família (Tabela 1 ).

Tabela 1 - Perfil sociodemográfico dos pais/responsáveis, Araçatuba, 2014

\begin{tabular}{|c|c|c|}
\hline Variáveis & $\mathrm{n}$ & $\%$ \\
\hline \multicolumn{3}{|l|}{ Sexo } \\
\hline Feminino & 89 & 60,5 \\
\hline Masculino & 58 & 39,5 \\
\hline \multicolumn{3}{|l|}{ Idade } \\
\hline 18 a 34 anos & 97 & 69,4 \\
\hline 35 a 44 anos & 33 & 22,4 \\
\hline 45 anos ou mais & 12 & 8,2 \\
\hline \multicolumn{3}{|l|}{ Cor/ raça } \\
\hline Branco & 67 & 45,6 \\
\hline Negro & 17 & 11,6 \\
\hline Pardo & 50 & 34,0 \\
\hline Amarelo & 5 & 3,4 \\
\hline Indígena & 1 & 0,7 \\
\hline Não respondeu & 7 & 4,8 \\
\hline \multicolumn{3}{|l|}{ Estado civil } \\
\hline Solteiro(a) & 55 & 37,4 \\
\hline Casado(a) & 68 & 46,3 \\
\hline União consensual & 15 & 10,2 \\
\hline Separado(a) & 3 & 2,0 \\
\hline Não respondeu & 6 & 4,1 \\
\hline \multicolumn{3}{|l|}{ Grau de instrução } \\
\hline Analfabeto & 2 & 1,4 \\
\hline 9 anos ou menos de estudo & 29 & 19,7 \\
\hline 10 a 12 anos de estudo & 83 & 56,5 \\
\hline Mais de 12 anos de estudo & 25 & 17,0 \\
\hline Não respondeu & 8 & 5,4 \\
\hline \multicolumn{3}{|l|}{ Tipo de vínculo trabalhista } \\
\hline Trabalha & 101 & 68,7 \\
\hline Não trabalha & 22 & 15,0 \\
\hline Não respondeu & 24 & 16,3 \\
\hline \multicolumn{3}{|l|}{ Renda familiar } \\
\hline Menos de 1 SM & 13 & 8,8 \\
\hline De 1 a 2 SM & 75 & 51,0 \\
\hline De 3 a 4 SM & 41 & 27,9 \\
\hline 5 ou mais SM & 8 & 5,5 \\
\hline Não respondeu & 10 & 6,8 \\
\hline \multicolumn{3}{|l|}{ Recebe Bolsa Família } \\
\hline Sim & 27 & 18,4 \\
\hline Não & 114 & 77,6 \\
\hline Não respondeu & 6 & 4,0 \\
\hline
\end{tabular}

Os escolares apresentaram o Ceod com média de 0,68 (desvio padrão $=1,70,0,52$ dentes cariados, $d p$ $=1,41$ e 0,16 dentes obturados, $d p=0,56$ ). A maioria das crianças apresentou um índice de higiene oral médio (IHO-S = 1,51, dp = 0,48) (Tabela 2).

Tabela 2 - Condição bucal dos escolares participantes do estudo, Araçatuba, 2014

\begin{tabular}{l|r|r|r|r|r}
\hline \multicolumn{1}{c|}{ Variáveis } & $\begin{array}{c}\text { Menor } \\
\text { valor }\end{array}$ & $\begin{array}{c}\text { Maior } \\
\text { valor }\end{array}$ & Mediana & Média & $\begin{array}{c}\text { Desvio } \\
\text { padrão }\end{array}$ \\
\hline Número de dentes & 8 & 24 & 20,00 & 17,99 & 3,99 \\
Cariados & 0 & 9 & 0 & 0,52 & 1,41 \\
Perdidos & 0 & 0 & - & - & - \\
Obturados & 0 & 3 & 0 & 0,16 & 0,56 \\
Ceod & 0 & 10 & 0 & 0,68 & 1,70 \\
IHO-S & 0,33 & 2,66 & 1,50 & 1,51 & 0,48 \\
\hline
\end{tabular}

Fonte: dados da pesquisa.

Em relação ao conhecimento, poucos pais conheciam problemas bucais como: periodontite $(27,9 \%)$, fluorose $(18,4 \%)$, fendas/fissuras (15\%) e má oclusão $(11,6 \%)$.

Apesar de 98,6\% acreditarem que a cárie é uma doença, 71,4\% achavam que ela não era uma doença transmissível. Quando perguntados sobre a frequência ideal de escovação, $57,8 \%$ responderam "após as refeições", e sobre o uso de fio dental, 28,6\% acreditavam que só deveriam usá-lo quando existissem alimentos entre os dentes (Tabela 3 ).

Já em relação à quantidade de creme dental para a escovação, $43,5 \%$ achavam necessário cobrir toda a escova com creme dental. Quanto ao dente de leite cariado, 33,3\% não sabiam se deveria ou não ser restaurado. Quando perguntados sobre o uso prolongado de chupeta, $93,2 \%$ sabiam que o uso prolongado é prejudicial, mas apenas $24,5 \%$ sabiam a idade de desuso (Tabela 3 ). 


\begin{tabular}{|c|c|c|}
\hline $\begin{array}{ll} & \text { Variáveis } \\
\end{array}$ & $n$ & $\%$ \\
\hline \multicolumn{3}{|c|}{ Q1- Na sua opinião, quais doenças podem ocorrer na boca? } \\
\hline Cárie & 145 & 98,6 \\
\hline Gengivite & 130 & 88,4 \\
\hline Câncer de boca & 118 & 80,3 \\
\hline Herpes & 99 & 67,3 \\
\hline Periodontite & 41 & 27,9 \\
\hline Fluorose & 27 & 18,4 \\
\hline Fendas/fissuras & 22 & 15,0 \\
\hline Má oclusão & 17 & 11,5 \\
\hline \multicolumn{3}{|l|}{ Q2- Você acha que cárie é uma doença transmissível? } \\
\hline Sim & 41 & 27,9 \\
\hline Não & 86 & 58,5 \\
\hline Não sabe & 19 & 12,9 \\
\hline \multicolumn{3}{|l|}{ Q3- O que você acha que causa a cárie dentária? } \\
\hline Não escovar os dentes & 146 & 99,3 \\
\hline Comer muito doce & 121 & 82,3 \\
\hline Não usar fio dental & 114 & 77,6 \\
\hline Não ir ao dentista & 90 & 61,2 \\
\hline Uso de antibiótico & 44 & 29,9 \\
\hline \multicolumn{3}{|l|}{ Q4- O que você acha preciso para prevenir a cárie? } \\
\hline Escovar os dentes diariamente & 145 & 98,6 \\
\hline Consultar o dentista & 127 & 86,4 \\
\hline Usar fio dental diariamente & 116 & 78,9 \\
\hline Não comer muito doce & 114 & 77,6 \\
\hline Ter uma alimentação saudável & 99 & 67,3 \\
\hline Aplicação de flúor & 83 & 56,5 \\
\hline Utilizar bochecho antes de dormir & 58 & 39,5 \\
\hline \multicolumn{3}{|c|}{ Q5- Qual a frequência que você acha ideal para escovação? } \\
\hline Uma vez ao dia & 5 & 3,4 \\
\hline Duas vezes ao dia & 5 & 3,4 \\
\hline Três vezes ou mais & 51 & 34,7 \\
\hline Após as refeições & 85 & 57,8 \\
\hline \multicolumn{3}{|c|}{ Q6- Qual a frequência que você acha ideal para o uso do fio dental? } \\
\hline Uma vez ao dia, de preferência à noite & 20 & 13,6 \\
\hline Duas ou mais vezes por dia & 82 & 55,8 \\
\hline Quando existem alimentos entre os dentes & 42 & 28,5 \\
\hline \multicolumn{3}{|c|}{ Q7- Qual a quantidade que você acha ideal de creme dental para a escovação? } \\
\hline Tamanho de um grão de ervilha & 62 & 42,2 \\
\hline Cobrir toda a escova & 64 & 43,5 \\
\hline Produção de espuma & 3 & 2,0 \\
\hline Não sei & 16 & 10,9 \\
\hline \multicolumn{3}{|c|}{ Q8- Quando você acha que é o momento ideal para primeira consulta ao dentista? } \\
\hline Antes do nascimento dos dentes & 77 & 52,4 \\
\hline Assim que nascer os primeiros dentes (6 meses) & 43 & 29,3 \\
\hline Dentição de leite completa (2 anos) & 17 & 11,6 \\
\hline Só quando a criança tiver cárie/dor de dente & 1 & 0,7 \\
\hline Não sabe & 9 & 6,1 \\
\hline \multicolumn{3}{|c|}{ Q9- Qual deve ser o intervalo de tempo em que a criança consulte o dentista? } \\
\hline De 3 em 3 meses & 67 & 45,6 \\
\hline De 6 em 6 meses & 67 & 45,6 \\
\hline De 1 ou 2 vezes ao ano & 9 & 6,1 \\
\hline Só quando estiver com dor & 1 & 0,7 \\
\hline \multicolumn{3}{|c|}{ Q10- Você acha que dente de leite cariado deve ser restaurado? } \\
\hline Sim & 75 & 51,0 \\
\hline Não & 20 & 13,6 \\
\hline Não sabe & 49 & 33,3 \\
\hline \multicolumn{3}{|c|}{ Q11- Você acha que o uso prolongado da chupeta é prejudicial para criança? } \\
\hline Sim & 137 & 93,2 \\
\hline Não & 4 & 2,7 \\
\hline Não sabe & 3 & 2,0 \\
\hline \multicolumn{3}{|c|}{ Q12- Qual idade você acha que a criança tem que abandonar a chupeta? } \\
\hline 1 ano & 79 & 53,7 \\
\hline 3 anos & 36 & 24,5 \\
\hline 6 anos & 2 & 1,4 \\
\hline Não sabe & 25 & 17,0 \\
\hline
\end{tabular}


$\mathrm{Na}$ análise bivariada, poucas associações estatisticamente significantes foram encontradas entre o conhecimento dos pais e as variáveis sociodemográficas. Houve associação estatisticamente significante entre a questão "o que causa cárie dentária" e a variável sexo dos pais. Associou-se a questão "o que é preciso para prevenir a cárie" com idade e raça dos pais (Tabela 4). A questão sobre a quantidade ideal de creme dental para a escovação esteve associada ao grau de instrução dos pais (Tabela 5).

Tabela 4 - Análise bivariada entre as variáveis sociodemográficas (independentes) e o conhecimento dos pais (dependente), Araçatuba, 2014

\begin{tabular}{|c|c|c|c|c|c|c|c|c|c|c|c|c|}
\hline \multirow{3}{*}{$\begin{array}{l}\text { Variáveis } \\
\text { Sexo }\end{array}$} & \multicolumn{12}{|c|}{ Conhecimento dos pais } \\
\hline & \multicolumn{2}{|c|}{ Q1 } & \multicolumn{2}{|c|}{ Q2 } & \multicolumn{2}{|c|}{ Q3 } & \multicolumn{2}{|c|}{ Q4 } & \multicolumn{2}{|c|}{ Q5 } & \multicolumn{2}{|c|}{ Q6 } \\
\hline & & & & & & & & & & & & \\
\hline Feminino & 87 & 2 & 29 & 60 & 32 & 57 & 71 & 18 & 52 & 36 & 56 & 30 \\
\hline Masculino & 56 & 0 & 11 & 45 & 11 & 45 & 42 & 14 & 31 & 25 & 44 & 12 \\
\hline p-valor & \multicolumn{2}{|c|}{$0,523^{*}$} & \multicolumn{2}{|c|}{0,090} & \multicolumn{2}{|c|}{0,036} & \multicolumn{2}{|c|}{0,500} & \multicolumn{2}{|c|}{0,658} & \multicolumn{2}{|c|}{0,086} \\
\hline \multicolumn{13}{|l|}{ Idade do pai } \\
\hline 18 a 34 anos & 97 & 0 & 27 & 70 & 28 & 69 & 81 & 16 & 53 & 43 & 68 & 27 \\
\hline 35 a 44 anos & 27 & 1 & 8 & 20 & 8 & 20 & 18 & 10 & 18 & 10 & 17 & 10 \\
\hline 45 anos ou mais & 9 & 1 & 2 & 8 & 3 & 7 & 6 & 4 & 7 & 3 & 7 & 3 \\
\hline p-valor & \multicolumn{2}{|c|}{$0,058^{* *}$} & \multicolumn{2}{|c|}{$0,851^{* *}$} & \multicolumn{2}{|c|}{0,996} & \multicolumn{2}{|c|}{$0,045^{* *}$} & \multicolumn{2}{|c|}{0,509} & \multicolumn{2}{|c|}{0,691} \\
\hline \multicolumn{13}{|l|}{ Cor/ raça } \\
\hline Branco & 66 & 1 & 17 & 50 & 19 & 48 & 19 & 48 & 37 & 30 & 51 & 15 \\
\hline Negro & 17 & 0 & 2 & 15 & 4 & 13 & 10 & 7 & 11 & 5 & 11 & 5 \\
\hline Pardo & 49 & 1 & 18 & 32 & 18 & 32 & 26 & 23 & 28 & 22 & 30 & 19 \\
\hline p-valor & 0,74 & & 0,1 & & 0,5 & & & & & & 0,1 & \\
\hline Estado civil & & & & & & & & & & & & \\
\hline Solteiro(a) & 55 & 0 & 13 & 42 & 17 & 38 & 45 & 10 & 29 & 26 & 39 & 14 \\
\hline Casado(a) & 66 & 2 & 19 & 49 & 19 & 49 & 49 & 19 & 39 & 29 & 46 & 22 \\
\hline União consensual & 15 & 0 & 6 & 9 & 4 & 11 & 12 & 3 & 11 & 3 & 10 & 4 \\
\hline p-valor & 0,23 & & 0,4 & & & & & & & & 0,7 & \\
\hline Grau de instrução & & & & & & & & & & & & \\
\hline Analfabeto & 2 & 0 & 0 & 2 & - & 2 & 1 & 1 & 2 & 0 & 2 & 0 \\
\hline 9 anos ou $<$ & 29 & 0 & 7 & 22 & 7 & 22 & 25 & 4 & 15 & 14 & 20 & 8 \\
\hline 10 a 12 anos & 81 & 2 & 22 & 61 & 28 & 55 & 64 & 19 & 49 & 34 & 56 & 26 \\
\hline Mais de 12 anos & 25 & 0 & 11 & 14 & 8 & 17 & 17 & 8 & 13 & 11 & 16 & 8 \\
\hline p-valor & 1,0 & & 0,2 & & 0,4 & & 0,3 & & 0,4 & & 0,65 & \\
\hline Vínculo trabalhista & & & & & & & & & & & & \\
\hline Trabalha & 99 & 2 & 30 & 71 & 27 & 74 & 79 & 22 & 55 & 45 & 70 & 29 \\
\hline Não trabalha & 22 & - & 4 & 18 & 4 & 18 & 15 & 7 & 9 & 13 & 14 & 8 \\
\hline $\mathrm{p}$-valor & 1,0 & & & & & & & & & & 0,5 & \\
\hline Renda familiar & & & & & & & & & & & & \\
\hline Menos de $1 \mathrm{SM}$ & 13 & 0 & 3 & 10 & 2 & 11 & 13 & - & 4 & 9 & 10 & 3 \\
\hline De 1 a 2 SM & 74 & 1 & 19 & 56 & 25 & 50 & 56 & 19 & 44 & 30 & 52 & 20 \\
\hline De 3 a 4 SM & 40 & 1 & 13 & 28 & 12 & 29 & 31 & 10 & 24 & 17 & 25 & 16 \\
\hline 5 ou mais SM & 8 & 0 & 4 & 4 & 3 & 5 & 5 & 3 & 5 & 3 & 7 & 1 \\
\hline p-valor & 1,0 & & 0,4 & & 0,5 & & & & 0,2 & & 0,33 & $* *$ \\
\hline Bolsa Família & & & & & & & & & & & & \\
\hline Sim & 27 & - & 5 & 22 & 6 & 21 & 22 & 5 & 12 & 14 & 19 & 6 \\
\hline Não & 112 & 2 & 34 & 80 & 36 & 78 & 87 & 27 & 68 & 46 & 78 & 35 \\
\hline p-valor & & $00^{*}$ & &, 238 & &, 339 & &, 564 & &, 210 & &, 490 \\
\hline
\end{tabular}

* Teste exato de Fisher.

** Razão de verossimilhança.

Fonte: dados da pesquisa. 
Tabela 5 - Análise bivariada entre as variáveis sociodemográficas (independentes) e o conhecimento dos pais (dependente), Araçatuba, 2014

\begin{tabular}{|c|c|c|c|c|c|c|c|c|c|c|c|c|}
\hline \multirow{3}{*}{ Variáveis } & \multicolumn{12}{|c|}{ Conhecimento dos pais } \\
\hline & \multicolumn{2}{|c|}{ Q7 } & \multicolumn{2}{|c|}{ Q8 } & \multicolumn{2}{|c|}{ Q9 } & \multicolumn{2}{|c|}{ Q10 } & \multicolumn{2}{|c|}{ Q11 } & \multicolumn{2}{|c|}{ Q12 } \\
\hline & & & & & & & & & & & & \\
\hline Feminino & 36 & 52 & 47 & 42 & 45 & 42 & 45 & 44 & 83 & 4 & 22 & 64 \\
\hline Masculino & 25 & 31 & 28 & 28 & 30 & 25 & 28 & 28 & 52 & 3 & 13 & 41 \\
\hline $\mathrm{p}$-valor & \multicolumn{2}{|c|}{0,658} & \multicolumn{2}{|c|}{0,742} & \multicolumn{2}{|c|}{0,743} & \multicolumn{2}{|c|}{0,947} & \multicolumn{2}{|c|}{$1,000^{*}$} & \multicolumn{2}{|c|}{0,841} \\
\hline \multicolumn{13}{|l|}{ Idade do pai } \\
\hline 18 a 34 anos & 36 & 60 & 48 & 49 & 52 & 42 & 53 & 44 & 88 & 7 & 25 & 69 \\
\hline 35 a 44 anos & 15 & 13 & 17 & 11 & 13 & 15 & 14 & 14 & 27 & - & 3 & 23 \\
\hline 45 anos ou mais & 5 & 5 & 6 & 4 & 4 & 6 & 3 & 7 & 10 & - & 4 & 6 \\
\hline p-valor & \multicolumn{2}{|c|}{0,272} & \multicolumn{2}{|c|}{0,513} & \multicolumn{2}{|c|}{0,513} & \multicolumn{2}{|c|}{0,324} & \multicolumn{2}{|c|}{ - } & \multicolumn{2}{|c|}{0,144} \\
\hline \multicolumn{13}{|l|}{ Cor/ raça } \\
\hline Branco & 31 & 36 & 39 & 28 & 37 & 28 & 36 & 31 & 63 & 3 & 22 & 44 \\
\hline Negro & 4 & 13 & 8 & 9 & 9 & 8 & 8 & 9 & 16 & 1 & 3 & 14 \\
\hline Pardo & 21 & 29 & 25 & 25 & 22 & 27 & 26 & 24 & 46 & 2 & 8 & 38 \\
\hline p-valor & & & 0,5 & & 0, & & & & 0,9 & & & \\
\hline Estado civil & & & & & & & & & & & & \\
\hline Solteiro(a) & 25 & 30 & 32 & 23 & 27 & 26 & 29 & 26 & 51 & 2 & 13 & 40 \\
\hline Casado(a) & 30 & 38 & 34 & 34 & 35 & 32 & 33 & 35 & 63 & 4 & 18 & 48 \\
\hline União consensual & 4 & 11 & 6 & 9 & 10 & 5 & 7 & 8 & 14 & 1 & 3 & 11 \\
\hline $\mathrm{p}$-valor & & & 0, & & 0,5 & & & & 0,8 & & & \\
\hline Grau de instrução & & & & & & & & & & & & \\
\hline Analfabeto & 2 & - & 2 & - & - & 2 & 1 & 1 & 2 & - & - & 2 \\
\hline 9 anos ou $<$ & 10 & 19 & 15 & 14 & 12 & 16 & 17 & 12 & 26 & 2 & 5 & 23 \\
\hline 10 a 12 anos & 42 & 41 & 46 & 37 & 48 & 33 & 40 & 43 & 78 & 3 & 23 & 56 \\
\hline Mais de 12 anos & 5 & 20 & 9 & 16 & 13 & 12 & 12 & 13 & 23 & 2 & 7 & 18 \\
\hline p-valor & 0,0 & & 0,1 & & 0,1 & & 0,7 & & 0,7 & & 0,4 & \\
\hline Vínculo trabalhista & & & & & & & & & & & & \\
\hline Trabalha & 42 & 59 & 52 & 49 & 56 & 44 & 48 & 53 & 95 & 5 & 25 & 73 \\
\hline Não trabalha & 10 & 12 & 11 & 11 & 11 & 9 & 10 & 12 & 19 & 1 & 4 & 16 \\
\hline p-valor & & & & & 0, & & & & 1,0 & & 0,7 & \\
\hline Renda familiar & & & & & & & & & & & & \\
\hline Menos de 1 SM & 5 & 8 & 9 & 4 & 6 & 7 & 7 & 6 & 13 & - & 3 & 10 \\
\hline De 1 a 2 SM & 31 & 44 & 37 & 38 & 37 & 35 & 37 & 38 & 68 & 4 & 17 & 53 \\
\hline De 3 a 4 SM & 21 & 20 & 22 & 19 & 24 & 17 & 22 & 19 & 39 & 2 & 11 & 30 \\
\hline 5 ou mais SM & 2 & 6 & 5 & 3 & 6 & 2 & 5 & 3 & 7 & 1 & 3 & 5 \\
\hline $\mathrm{p}$-valor & 0,4 & & 0,5 & & 0,5 & & 0,8 & & 0,5 & & 0,8 & \\
\hline Bolsa Família & & & & & & & & & & & & \\
\hline Sim & 11 & 16 & 16 & 11 & 15 & 11 & 15 & 12 & 26 & - & 4 & 22 \\
\hline Não & 49 & 65 & 59 & 55 & 59 & 53 & 56 & 58 & 105 & 7 & 32 & 78 \\
\hline p-valor & & & & & & & & & 0,3 & & & \\
\hline
\end{tabular}

* Teste exato de Fisher.

** Razão de verossimilhança.

Fonte: dados da pesquisa. 


\section{Discussão}

A avaliação da condição socioeconômica permite considerar possíveis fatores etiológicos das desigualdades sociais no Brasil, como renda, níveis de escolaridade e condições de moradia. Nesse sentido, verificou-se que grande parte dos pais participantes do estudo vivia com uma renda de um a dois salários, o que é considerado de média baixa renda ${ }^{18}$. Fatores socioeconômicos podem ter influência na saúde bucal das crianças ${ }^{19}$, sendo que, neste estudo, a maioria dos pais das crianças tinha escolaridade entre 10 e 12 anos, o que pode ter contribuído com os resultados positivos para baixa prevalência de cárie.

A condição de saúde bucal das crianças participantes do estudo era boa, sendo a média do Ceod menor que os resultados encontrados na pesquisa de abrangência nacional realizada pelo Ministério da Saúde brasileiro, a qual recebeu o título de Projeto SBBrasil $2010^{9}$, em que $79,6 \%$ da amostra estava livre de cárie. Esses resultados corroboram com outras pesquisas recentes realizadas no Brasil, que encontraram que a minoria das crianças apresentava experiência de cárie $\mathrm{e}^{19-21}$.

A condição de saúde bucal das crianças é, tradicionalmente, associada ao conhecimento sobre saúde bucal de seus pais e responsáveis, já que os hábitos relacionados à higiene oral e à dieta são estabelecidos durante a infância e mantidos ao longo dessa fase ${ }^{22}$. No entanto, no presente estudo, não foi observado tal fato, já que a condição bucal das crianças avaliadas era boa, enquanto o conhecimento dos pais sobre as doenças que podem acometer a cavidade oral, a transmissibilidade da cárie e os fatores etiológicos da cárie dentária não foi satisfatório.

A maioria dos pais não tinha conhecimento sobre doença periodontal $(72,1 \%)$, fluorose dentária $(81,6 \%)$ e má oclusão $(88,4 \%)$. Além disso, $58,5 \%$ afirmaram que a cárie não é uma doença transmissível - apesar do fato de que o compartilhamento de utensílios pode transmitir Streptococus mutans e causar cárie em crianças -, o que é semelhante ao relatado em um estudo realizado por Sakai et al. ${ }^{23}$ (2008). O modo de transmissão pode ocorrer por via direta ou indireta, sendo o ato de beijar o contato direto mais comum, de modo que a flora oral é transmitida na saliva, e o contato indireto ocorre por meio de objetos, como copo, utensílios, escovas de dente, ou até mesmo brinquedos comuns, que são contaminados com bactérias cariogênicas ${ }^{24-26}$.

Observa-se que os entrevistados têm pouco conhecimento sobre a importância dos dentes decíduos. Muitos pais disseram não saber ou não ser necessário o tratamento de dentes decíduos cariados, tal resultado também foi observado em outros estudos $^{26-28}$. Esse fato é preocupante, pois muitas são as funções dos elementos que compõem a dentição decídua, os quais devem ser mantidos em condições adequadas na cavidade bucal até a sua esfoliação ${ }^{29}$.

A atenção odontológica precoce é uma das ferramentas mais eficientes contra a cárie dentária e outros problemas bucais ${ }^{30}$. Em relação ao momento adequado para a primeira visita ao dentista, grande parte apresentou conhecimento adequado, pois $52,4 \%$ dos entrevistados acreditam que essa deve ocorrer antes que os elementos dentais irrompam. No entanto, a Academia Americana de Pediatria recomenda que todas as crianças devem começar a receber avaliações de risco em saúde bucal com 6 meses de idade por um profissional de saúde qualificado ${ }^{31}$.

Os entrevistados deste estudo consideraram o uso prolongado da chupeta prejudicial à criança, entretanto, poucos demonstraram conhecimento sobre a idade limite para o seu abandono (até 3 anos), período no qual o desenvolvimento facial pode ser comprometido e alterações na morfologia das arcadas dentárias podem ser percebidas, como mordida aberta anterior e cruzada ${ }^{32}$.

$\mathrm{Na}$ análise bivariada, associações significativas foram observadas entre o conhecimento dos pais e as variáveis sociodemográficas, sendo que o sexo dos pais esteve relacionado ao maior conhecimento sobre as causas da cárie dentária. Isso pode ser explicado pelo fato de que as mulheres (mães) apresentam uma ligação afetiva maior com a criança e um papel mais forte como educadora e cuidadora, além de estarem mais interessadas em buscar informações ${ }^{33}$. A idade esteve associada com maior conhecimento sobre prevenção da cárie, sendo que os pais mais jovens têm, geralmente, maior acesso aos meios de comunicação (computador/internet) e, portanto, mais informação relacionada à saúde oral. No entanto, muitos pais mais jovens estão ocupados com sua carga horária de trabalho, deixando em segundo plano as responsabilidades familiares, as quais não são vistas como prioridade, de modo que seus filhos contam com o apoio da escola no cuidado diário e na educação ${ }^{34}$.

Assim como nos estudos realizados por Suresh et al. ${ }^{22}$ (2010) e Williams et al. ${ }^{35}$ (2002), também foi observado, nesta pesquisa, que os pais com baixa escolaridade tinham baixo nível de conhecimento sobre higiene oral. Isso acontece pelo fato de que os pais com maior nível de escolaridade são mais propensos a ter atitudes positivas de saúde e a prestar uma maior atenção à saúde da criança ${ }^{36}$.

\section{Conclusão}

Conclui-se que o conhecimento dos pais e responsáveis sobre saúde bucal não se mostrou adequado, poucos conheciam alguns os problemas bucais, o modo de transmissão da cárie, as indicações e modo de usar creme dental e fio dental. Mesmo com a falta de conhecimento, os filhos apresenta- 
vam uma boa condição de saúde bucal. Fatores sociodemográficos como sexo, idade, raça e grau de instrução dos pais estiveram associadas ao maior conhecimento sobre saúde bucal. Assim, é necessário maior atenção a esse grupo, com realização de atividades educativas voltadas para a capacitação dos pais/responsáveis, para que atuem de forma direta na manutenção da saúde bucal de seus filhos juntamente com os projetos realizados nas escolas.

\section{Abstract}

Objective: To assess the oral health status of preschoolers and the knowledge of parents on oral health. Subjects and method: Cross-sectional analytical study with a sample of 147 parents and their children, in public schools of basic education of Araçatuba, SP, Brazil. A questionnaire with sociodemographic and oral health knowledge questions was applied. Oral clinical examination was performed in children to verify dmft and OHI-S. Data were analyzed using the SPSS software. Results: The majority of respondents were women $(60.5 \%)$ aged $18-34$ years (66.0\%). Regarding level of education, $56.5 \%$ studied from 10 to 12 years and $68.7 \%$ worked, but the majority had family income between 1 and 2 minimum wages (51\%) and $18.4 \%$ received family allowance. The oral health status of children was good and the average dmft was 0.68. Most children presented mean oral hygiene index (OHI-S 1.51, sd=0.48). Bivariate analysis showed an association between the cause of dental caries and gender of parents $(p=0.036)$. Caries prevention was statistically associated with age $(p=0.045)$ and race $(p=0.008)$ of parents. Conclusion: Knowledge of parents and guardians on oral health was not adequate, despite the good condition of their children's oral health. Sociodemographic factors such as gender, age, race, and level of education of parents were associated with greater knowledge on oral health.

Keywords: Health education. Knowledge. Oral health. Preschool.

\section{Referências}

1. Antunes LS, Soares MB, Antunes LA, Corvino MPF. Conhecimentos, práticas e atitudes de responsáveis frente à saúde bucal do pré-escolar. Odontol Clín-Científ 2008; 7(3):241-6.

2. Nakre PD, Harikiran AG. Effectiveness of oral health education programs: a systematic review. J Int Soc Prevent Communit Dent 2013; 3(2):103-15.

3. Gauba A, Bal IS, Jain A, Mittal HC. School based oral health promotional intervention: Effect on knowledge, practices and clinical oral health related parameters. Contemp Clin Dent 2013; 4:493-9.

4. Sheiham A, Watt R. Oral health promotion and policy. In: Murray JJ, Nunn JH, Steele JG, editors. Prevention of Oral Disease. Oxford: Oxford University Press; 2003. p. 241-58.

5. Figueira TR, Leite ICG. Percepção, conhecimento e práticas em saúde bucal de escolares. RGO 2008; 56(1):27-32.

6. Fuller LA, Stull SC, Darby ML, Tolle SL. Oral health promotion: knowledge, confidence, and practices in preventing early-severe childhood caries of Virginia WIC Program Personnel. J Dent Hyg 2014; 88(2):130-40.
7. Weyne SC, Harari SG. Cariologia: implicações e aplicações clínicas. In: Baratieri LN. Odontologia restauradora: fundamentos e possibilidades. São Paulo: Editora Santos; 2001. p. 3-26.

8. Gambhir RS, Sohi RK, Nanda T, Sawhney GS, Setia S. Impact of school based oral health education programmes in India: a systematic review. J Clin Diagn Res 2013; 7(12):3107-10.

9. Projeto SB Brasil 2010. Pesquisa Nacional de Saúde Bucal - resultados principais. Brasília: Ministério da Saúde; 2011.

10. Campos L, Bottan ER, Birolo JB, Silveira EG, Schmitt BHE. Conhecimento de mães de diferentes classes sociais sobre saúde bucal no município de Cocal do Sul (SC). Rev Sul-Bras Odontol 2010; 7(3):2872-95.

11. Flanders RA. Eficácia dos programas de educação para a saúde dental nas escolas. J Am Dent Assoc 1987; 114:239-42.

12. Pine CM. Designing school programmes to be effective vehicles for changing oral hygiene behaviour. Int J Dent 2007; 57:377-81.

13. Guimarães AO, Costa ICC, Oliveira ALS. As origens, objetivos e razões de ser da odontologia para bebês. J Bras Odontoped Odontol Bebê 2003; 6(29):83-6.

14. Garbin CAS, Garbin AJI, Dos Santos KT, Lima DP. Oral health education in schools: promoting health agents. Int $J$ Dent Hyg. 2009; 7(3):212-6.

15. Massoni ACLT, Forte FDS, Sampaio FC. Percepção de pais e responsáveis sobre promoção de saúde bucal. Rev Odontol Unesp 2005; 34(4):193-7.

16. World Health Organization. Oral health surveys: basic methods. 4. ed. Geneva: World Health Organization; 1997.

17. Greene JC, Vermillion JR. The simplified oral hygiene index. J Amer Dent Ass 1964; 68:25-31.

18. Marmot M. Social determinants of health inequalities. The Lancet 2005; 365:1099-104.

19. Bonanato K, Pordeus IA, Moura-Leite FR, Ramos-Jorge ML, Vale MP, Paiva SM. Oral disease and social class in a random sample of five-year-old preschool children in a Brazilian city. Oral Health Prev Dent 2010; 8:125-32.

20. Paula JS, Leite IC, Almeida AB, Ambrosano GM, Pereira AC, Mialhe FL. The influence of oral health conditions, socioeconomic status and home environment factors on schoolchildren's self-perception of quality of life. Health Qual Life Outcomes 2012; 10:6.

21. Martins MT, Sardenberg F, Abreu MH, Vale MP, Paiva SM, Pordeus IA. Factors associated with dental caries in Brazilian children: a multilevel approach. Community Dent Oral Epidemiol 2013; 42(4):289-99.

22. Suresh BS, Ravishankar TL, Chaitra TR, Mohapatra AK, Gupta V. Mother's knowledge about pre-school child's oral health. J Indian Soc Pedod Prev Dent 2010; 28(4):282-7.

23. Sakai VT, Oliveira TM, Silva TC, Moretti ABS, Geller-Palti D, Biella VA, et al. Knowledge and attitude of parents or caretakers regarding transmissibility of caries disease. $\mathrm{J}$ Appl Oral Sci 2008; 16:150-4.

24. Keyes PH. The infectious and transmissible nature of experimental Dental Caries. Arch Oral Biol 1960; 1:304-20.

25. Chan KM, King NM, Kilpatri NM. Can infant catch caries? A review of the current evidence on the infectious nature of dental caries in infants. N Z Dent J 2005; 101:4-11.

26. Chhabra N, Chhabra A. Parental knowledge, attitudes and cultural beliefs regarding oral health and dental care of preschool children in an Indian population: a quantitative study. Eur Arch Paediatr Dent 2012; 13(2):76-82.

27. Prabhu A, Rao AP, Reddy V, Ahamed SS, Muhammad S, Thayumanavan S. Parental knowledge of pre-school child oral health. Community Health 2013; 38(5):880-4. 
28. Szatko F, Wierzbicka M, Dybizbanska E, Struzycka I, Iwanicka-Frankowska E. Oral health of polish three-year olds and mother's oral health-related knowledge. Community Dent Health 2004; 21:175-80.

29. Massoni ACLT, Paulo SF, Forte FDS, Freitas CHSM, Sampaio FC. Saúde bucal infantil: conhecimento e interesse de pais e responsáveis. Pesq Bras Odontoped Clin Integr 2010; 10(2):257-64.

30. Moura LFAD, MouraI MS, Toledo OA. Conhecimentos e práticas em saúde bucal de mães que frequentaram um programa odontológico de atenção materno-infantil. Cien Saúde Colet 2007; 12(4):1079-86.

31. American Academy of Pediatrics. Oral health risk assessment timing and establishment of the dental home. Pediatrics 2003; 111(5):1113-6.

32. Charchut SW. The effects of infant feeding on the occlusion of the primary dentition. J Dent Child 2003; 3(7):197-203.

33. Wilson A, Brega AG, Batliner TS, Henderson W, Campagna EJ, Fehringer K, et al. Assessment of parental oral health knowledge and behaviors among American Indians of a Northern Plains tribe. Public Health Dent 2014; 74:159-67.

34. Ashkanani F, Al-sane M. Knowledge, attitudes and practices of caregivers in relation to oral health of preschool children. Med Princ Pract 2013; 22(2):167-72.

35. Williams NJ, Whittle JG, Gatrell AC. The relationship between socio-demographic characteristics and dental health knowledge and attitudes of parents with young children. $\mathrm{Br}$ Dent J 2002; 193:651-4.

36. Nagarajappa R, Kakatkar G, Sharda AJ, Asawa K, Ramesh G, Sandesh N. Infant oral health: knowledge, attitude and practices of parents in Udaipur, India. Dent Res J (Isfahan) $2013 ; 10(5): 659-65$.

\section{Endereço para correspondência:}

Gabriella Barreto Soares

Rua José Bonifácio,1193

Vila Mendonça

16015-050 Araçatuba, SP

Telefone: (18)3636-3249

E-mail: gabriella.barreto@yahoo.com.br

Recebido: 16/05/16. Aceito: 10/06/16. 